\title{
Infinite Differentiability \\ for One-Dimensional Spin System with Long Range Random Interaction
}

\author{
M. Cassandro ${ }^{1}$, E. Olivieri ${ }^{2}$, and B. Tirozzi ${ }^{2}$ \\ 1 Istituto di Física, Università di Roma, C.N.R. (G.N.S.M.), I-00185 Roma, Italy \\ 2 Istituto di Matematica, Università di Roma, C.N.R. (G.N.F.M.), I-00185 Roma, Italy
}

Abstract. We consider one-dimensional spin systems with Hamiltonian:

$$
H\left(\sigma_{\Lambda}\right)=-\sum_{t, t^{\prime} \in \Lambda} \frac{\varepsilon_{t t^{\prime}}}{\left|t-t^{\prime}\right|^{\alpha}} \sigma_{t} \sigma_{t^{\prime}}-h \sum_{t \in A} \sigma_{t},
$$

where $\varepsilon_{t^{\prime}}$ are independent random variables and, using decimation and the cluster expansion, we show that, when $\alpha>3 / 2$ and $\mathbb{E}\left(\varepsilon_{t t^{\prime}}\right)=0$, for any magnetic field $h$ and inverse temperature $\beta$, the correlation functions and the free energy are $C^{\infty}$ both in $h$ and $\beta$.

Moreover we discuss an example, obtained by a particular choice of the probability distribution of the $\varepsilon_{t t^{\prime}}$ 's, where the quenched magnetization is $C^{\infty}$ but fails to be analytic in $h$ for suitable $h$ and $\beta$.

\section{Introduction and Results}

We consider a one-dimensional system with random interaction enclosed in a box $\Lambda$ whose energy, for a given spin configuration $\sigma_{\Lambda}$ in $\Lambda$, is:

$$
H\left(\sigma_{\Lambda}\right)=-\sum_{\substack{t_{1}, t_{2} \in \Lambda \\ t_{1} \neq t_{2}}} \frac{\varepsilon_{t_{1} t_{2}}}{\left|t_{1}-t_{2}\right|^{\alpha}} \sigma_{t_{1}} \sigma_{t_{2}}-h \sum_{t \in \Lambda} \sigma_{t},
$$

where $\sigma_{t} \in\{1,-1\}, 3 / 2<\alpha<2^{1}$ and $\varepsilon_{t_{1} t_{2}}$ are independent random variables defined in the probability space $(\Omega, \Sigma, \mathbb{P})$.

In the sequel we will consider the following conditions on the $\varepsilon_{t_{1} t_{2}}$ :

C1) $\mathbb{E}\left(\varepsilon_{t_{1} t_{2}}\right)=0$,

C2) $\exists \bar{\varepsilon}:\left|\varepsilon_{t_{1} t_{2}}\right|<\bar{\varepsilon} \quad \forall t_{1}, t_{2} \in \mathbb{Z}$,

C3) $\mathbb{E}\left(\varepsilon_{t_{1} t_{2}}^{2}\right) \geqq a$ for some $a>0$,

C4) the probability distribution of $\varepsilon_{t_{1} t_{2}}$ depends only on $\left|t_{1}-t_{2}\right|$ (translation invariance).

1 For $\alpha>2$ the stochastic character of the interaction is irrelevant (see [1] and Remark 3 of Sect. 4) 
Calling $\omega \in \Omega$ a realization of a random field $\left\{\varepsilon_{t_{1}, t_{2}},\left(t_{1}, t_{2}\right) \in \mathbb{Z}\right\}$ we shall study the properties, as $\Lambda$ goes to infinity, of sequences of random variables as:

$$
\langle f\rangle_{\omega, \beta, h}^{\Lambda}=\frac{\sum_{\sigma_{\Lambda}} f\left(\sigma_{\Lambda}\right) \exp \left[-\beta H\left(\sigma_{A}\right)\right]}{\sum_{\sigma_{\Lambda}} \exp \left[-\beta H\left(\sigma_{\Lambda}\right)\right]},
$$

where $f$ is a cylindrical function or

$$
F_{\Lambda}(\omega, \beta, h)=\frac{1}{|\Lambda|} \log \sum_{\sigma_{\Lambda}} \exp \left[-\beta H\left(\sigma_{\Lambda}\right)\right] .
$$

We shall also consider the properties of their expectations:

$$
\begin{aligned}
\mathbb{E}\left(\langle f\rangle_{\cdot, \beta, h}^{\Lambda}\right) & =\int \mathbb{P}(d \omega)\langle f\rangle_{\omega, \beta, h}^{\Lambda}, \\
\mathbb{E}\left(F_{\Lambda}(\cdot, \beta, h)\right) & =\int \mathbb{P}(d \omega) F_{\Lambda}(\omega, \beta, h) .
\end{aligned}
$$

To state our results let us recall the definition of the space $\mathscr{E}\left(\mathbb{R}^{2}\right)$ : the space of $C^{\infty}$ functions on $\mathbb{R}^{2}$ with the topology given by the convergence of all derivatives uniformly on compact sets.

Theorem 1. Suppose conditions $\mathrm{C} 1, \mathrm{C} 2, \mathrm{C} 3$ are satisfied and consider an inclusive sequence of intervals invading $\mathbb{Z}$, then for any cylindrical function $f$ the quantity $\langle f\rangle_{\omega, \beta, h}^{\Lambda}$ converges $\mathbb{P}$ almost everywhere in $\mathscr{E}\left(\mathbb{R}^{2}\right)$.

Under the same hypotheses the quantity $\mathbb{E}\left(\langle f\rangle_{., \beta, h}^{\Lambda}\right)$ also converges in $\mathscr{E}\left(\mathbb{R}^{2}\right)$.

Theorem 2. Suppose conditions C1), C2), C3), C4) are satisfied and consider an inclusive sequence of intervals $\Lambda$ invading $\mathbb{Z}$, then:

i) The quantity $\mathbb{E}\left(F_{\Lambda}(\omega, \beta, h)\right)$ converges in $\mathscr{E}\left(\mathbb{R}^{2}\right)$ to a function $F(\beta, h)$.

ii) The quantity $F_{\Lambda}(\omega, \beta, h)$ converges $\mathbb{P}$-almost everywhere to $F(\beta, h)$ in $\mathscr{E}\left(\mathbb{R}^{2}\right)$.

Part of the results listed above was already known (cf. $[2,3]$ ), but the $C^{\infty}$ properties are new.

In [2] Khanin and Sinai prove that for a $v$-dimensional lattice system described by a Hamiltonian of the form (1.1), the thermodynamic limit for the free energy exists with probability one and it is equal to its average value, provided $\alpha>v / 2$.

In [3] Khanin considers the very same model we discuss in this paper and shows that, under the same conditions we assume for Theorem 1, the Gibbs states are unique at any temperature with probability one.

The new results on the $C^{\infty}$ properties are obtained by using the techniques introduced in [1] for interaction with finite first moment $(\alpha>2)$. This approach relies on the fact that when the interaction among two half lines at a distance $l$ is infinitesimal with $l$, then, for arbitrary $\beta$ and $h$, by grouping the spins in suitable blocks, it is possible to average over alternating blocks (decimation procedure) and obtain a weak effective interaction; then any quantity of relevance can be expressed in terms of a convergent series (cluster expansion). Our method consists in showing that with high probability a random system with $3 / 2<\alpha<2$ behaves very similarly to a regular system with $\alpha>2$. In fact by grouping the sites of $\mathbb{Z}$ in blocks whose size is increasing with the distance, say, from the origin, we are able 
to show that the typical interaction among noncontiguous blocks can be made sufficiently small to satisfy the requirements of [1]. We introduce for any given $\omega$ the set $C(\omega)$, which is the minimal interval, centered at the origin, containing all the "bad blocks" (i.e. all the blocks whose interaction with some nonadjacent block is not sufficiently small), and we show that $C(\omega)$ is finite with probability one.

If we consider now, say, $D^{p}\left\langle\sigma_{0}\right\rangle_{\omega, \beta, h}^{\Lambda}$ standard manipulations of the cluster expansion allow us to prove a bound, uniform in $\Lambda$, which behaves like $|\operatorname{diam} C|^{p}$. To extend these results to extensive quantities, some extra work is needed, because our partition of $\mathbb{Z}$ into blocks is not translationally invariant.

To study the free energy, for instance, we express its first derivatives as a sum of local quantities $\left(\frac{d F_{\Lambda}}{d h}=\frac{1}{|\Lambda|} \sum_{t \in \Lambda}\left\langle\sigma_{t}\right\rangle\right)$, and then we control this sum and its derivatives with the law of large numbers.

To conclude this section, we want to briefly discuss the analyticity properties of random systems. The structure of the series expansion we consider is such that each term is an analytic function both in $\beta$ and $h$ in a suitable neighborhood of the real axis, the position of the nearest singularity being related to the maximal size of the blocks involved. For the class of potentials studied in [1], due to the boundedness of the blocks, it was possible to prove, via Vitali's theorem, the analyticity in $\beta$ and $h$ of the limiting free energy in a finite neighborhood of the real axis.

For the class of potentials considered in this paper, infinite differentiability comes out to be a general property, but the increasing size of the blocks does not allow us to make any statement about analyticity.

The presence and the nature of the singularities in $\beta$ and $h$ have already been discussed for a class of random models by Griffiths and Lebowitz [4] and Griffiths [5]. They consider a dilute Ising model with nearest neighbour interaction, where $p$ is the probability for a site to be occupied. In [4] it is shown that, when $p$ and $\beta$ are sufficiently high, spontaneous magnetization is present. In [5] it is shown that the average magnetization is nonanalytic for $T<T_{c}$, where $T_{c}$ is the critical temperature of the corresponding ferromagnetic system. Furthermore, since for $p<p_{0}$ (where $p_{0}$ is the percolation threshold for the independent system of sites) the spontaneous magnetization is zero and the Gibbs state is unique (see Georgii [6]), it is natural to inquire to what extent nonanalyticity affects the differentiability properties of random systems.

In the last section of this paper we will exhibit a model described by a Hamiltonian of the form (1.1), where, by suitably choosing the probability distribution of the $\varepsilon$ 's, the limiting free energy is $C^{\infty}$ both in $\beta$ and $h$, but it fails to be analytic in $h$ at $h=0$, when $3 / 2<\alpha<2$ for any $T<T_{c}(\alpha)$.

\section{The Blocks and the Polymer Expansion}

Let us define a partition of $\mathbb{Z}$ into blocks whose size is logarithmically increasing with the distance from the origin. In the sequel we shall label the sites of the lattice with $t$ and the blocks with the index $n$. The positive values of $n$ correspond to the blocks on the right of the origin, the negative ones to the blocks on the left. 
Given an odd integer $q_{0}$ we call $Q(0)$ the interval centered at the origin with size $q_{0} . Q(1)$ is defined as

$$
Q(1) \equiv\left\{t \in \mathbb{Z}: \frac{q_{0}-1}{2}+1 \leqq t \leqq \frac{q_{0}-1}{2}+q(1)\right\},
$$

and for $n \geqq 2$

$$
Q(n) \equiv\left\{t \in \mathbb{Z}: \frac{q_{0}-1}{2}+1+\sum_{j=1}^{n-1} q(j) \leqq t \leqq \frac{q_{0}-1}{2}+\sum_{j=1}^{n} q(j)\right\} .
$$

For $n<0, n=-|n|$, we define $Q(-|n|)=-Q(|n|)$, i.e. the symmetric set with respect to the origin. For $n>0, q(n)=q_{0}\left[\left(\log _{+} n\right)^{0}\right] ;[\cdot]$ means integer part and $\log _{+} x$ $=\max (1, \log x)$. The increasing size of the blocks is necessary to control the fluctuations of the interaction energy all over $\mathbb{Z}$; but the actual form proportional to $\left(\log _{+} n\right)^{\varrho}$ is due to the following considerations:

i) $\varrho>2+\frac{2}{\alpha-3 / 2}$ is sufficient to get "good" probability estimates [cf. Eq. (2.15)],

ii) a power law will give rise to problems in estimating derivatives of arbitrary order. Now for any given $\omega$ we will define the "bad blocks" in such a way that, once they are collected in a set $C$, it will be possible to perform the cluster expansion outside $C$.

The probability estimate for having "strong" interactions among nonconsecutive blocks is based on Bernstein's inequality [7]: let $\eta_{i}, i=1,2, \ldots, n$ be independent random variables satisfying the following conditions:

$$
\mathbb{E} \eta_{i}^{k} \leqq \frac{1}{2} \mathbb{E} \eta_{i}^{2} \mathrm{~b}^{k-2} k ! \quad \forall k \geqq 2,
$$

where $b$ is a given positive number.

$$
\text { If } \begin{aligned}
D_{n}=\sum_{i=1}^{n} \mathbb{E} \eta_{i}^{2}, 0 \leqq & t \leqq \sqrt{D_{n}} / 2 b \text {, then } \\
& \mathbb{P}\left(\left|\sum_{i=1}^{n} \eta_{i}\right| \geqq 2 t \sqrt{D_{n}}\right) \leqq 2 \exp \left(-t^{2}\right) .
\end{aligned}
$$

Now if we denote by $W\left(\sigma_{V_{1}}, \sigma_{V_{2}}\right)$ the interaction energy

following $[2,3]$ we have :

$$
W\left(\sigma_{V_{1}}, \sigma_{V_{2}}\right)=\beta \sum_{\substack{t_{1} \in V_{1} \\ t_{2} \in V_{2}}} \varepsilon_{t_{1} t_{2}} \frac{\sigma_{t_{1}} \sigma_{t_{2}}}{\left|t_{1}-t_{2}\right|^{\alpha}}
$$

Lemma 2.1. Let conditions $\mathrm{C} 1, \mathrm{C} 2, \mathrm{C} 3$ be satisfied, then for any $\delta: 0<\delta<1 / 2$ and $q_{0}$ sufficiently large:

$$
\begin{aligned}
\mathbb{P}\left\{\exists \sigma_{Q\left(n_{1}\right)}, \sigma_{Q\left(n_{2}\right)}:\left|W\left(\sigma_{Q\left(n_{1}\right)}, \sigma_{Q\left(n_{2}\right)}\right)\right|\right. & \left.\geqq \frac{\text { const }}{q_{0}^{\lambda}\left(\log _{+} n_{2}\right)^{\varrho \varepsilon}\left|n_{2}-n_{1}\right|^{\alpha}}\right\} \\
& \leqq \text { const } \exp \left\{-q_{0}^{1+\delta}\left(\log _{+}\left|n_{2}\right|\right)^{\varrho}\right\},
\end{aligned}
$$

where $\varepsilon=\alpha-3 / 2, \lambda=\varepsilon-\delta$ and $\left|n_{2}\right|>\left|n_{1}\right|$. 
Proof. It follows from Bernstein's inequality by setting:

$$
\eta_{i j}=\varepsilon_{i j} \frac{\sigma_{i} \sigma_{j}}{|i-j|^{\alpha}}\left[d\left(n_{1}, n_{2}\right)\right]^{\alpha}
$$

$\left[d\left(n_{1}, n_{2}\right)\right.$ is the distance between the blocks $\left.Q\left(n_{1}\right), Q\left(n_{2}\right)\right]$, and choosing $t=q_{0}^{1 / 2+\delta}$ $\left(\log _{+}\left|n_{2}\right|\right)^{\varrho / 2}$. blocks :

Following [1] we introduce a partition of the lattice $\mathbb{Z}$ into alternating $A$ and $B$

$$
\mathbb{Z}=\bigcup_{n=-\infty}^{+\infty}(B(n) \cup A(n))
$$

where $A(n)=Q(2 n), B(n)=Q(2 n-1)$. We use the following notations for the spin configurations in the blocks

$$
\gamma_{n}=\sigma_{Q(n)}, \quad \alpha_{n}=\sigma_{A(n)}, \quad \beta_{n}=\sigma_{B(n)},
$$

and if $X$ is a set of $Q$-blocks we write $\gamma_{X}$ instead of $\left(\gamma_{n}\right)_{Q(n) \in X}$ (analogously for $\beta$ and $\alpha$ variables). From now on we will consider random spin systems enclosed in a box $A$, containing the origin, which is exactly partitioned into $A$ and $B$ blocks but not necessarily symmetric with respect to the origin. The box $\Lambda$ contains, say, the $Q$-blocks with index between $-2 m$ and $2 m^{\prime}$ so that $\Lambda$ starts and end with $A$-blocks.

Call

$$
\begin{aligned}
\Gamma(\omega) & =\left\{Q(n): \exists n^{\prime} \neq n \pm 1: \text { for some } \sigma_{Q(n)}, \sigma_{Q\left(n^{\prime}\right)} \mid W\left(\sigma_{Q(n)}, \sigma_{Q\left(n^{\prime}\right)} \mid\right.\right. \\
& \left.\geqq \frac{\text { const }}{q_{0}^{\varepsilon / 2}\left[\log _{+}\left(\max |n|,\left|n^{\prime}\right|\right)\right]^{\varrho \varepsilon}\left|n-n^{\prime}\right|^{\alpha}}\right\} .
\end{aligned}
$$

Consider now a block $B(n)$ and the spin configurations $\alpha_{n-1}, \alpha_{n}$ in the adjacent blocks $A(n), A(n-1)$, and let $Z_{B(n)}^{\alpha_{n}-1, \alpha_{n}}$ be the following partition function:

$$
\begin{aligned}
Z_{B(n)}^{\alpha_{n-1}, \alpha_{n}}= & \sum_{\sigma_{B(n)}} \exp \left\{\beta \sum_{t_{1} \neq t_{2} \in B(n)} \frac{\varepsilon_{t_{1} t_{2}} \sigma_{t_{1}} \sigma_{t_{2}}}{\left|t_{1}-t_{2}\right|^{\alpha}}\right\} \\
& \cdot \exp \left\{\beta \sum_{\substack{t_{1} \in B(n) \\
t_{2} \in A(n-1) \cup A(n)}} \varepsilon_{t_{1} t_{2} t_{2}} \frac{\sigma_{t_{1}} \sigma_{t_{2}}}{\left|t_{1}-t_{2}\right|^{\alpha}}+h \sum_{t \in B(n)} \sigma_{t}\right\} .
\end{aligned}
$$

Set $0=(-1,-1, \ldots,-1)$ a reference spin configuration, and let $\psi\left(\alpha_{n-1}, \alpha_{n}\right)$ be

$$
\psi\left(\alpha_{n-1}, \alpha_{n}\right)=\log \frac{Z_{B(n)}^{\alpha_{n-1}, \alpha_{n}} Z_{B(n)}^{0.0}}{Z_{B(n)}^{\alpha_{n}, 1,0} Z_{B(n)}^{0, \alpha_{n}}} .
$$

Call $\bar{\Gamma}(\omega)$ the set of all triples $A(n-1), B(n), A(n)$ such that

$$
\left|\psi\left(\alpha_{n-1}, \alpha_{n}\right)\right|>\frac{1}{\left[q_{n}\left(\log _{+} n\right)^{\varrho}\right]^{\varepsilon / 4}} \text { for some } \alpha_{n-1}, \alpha_{n} .
$$

Meanwhile the "bad" nature of the $Q$-blocks belonging to $\Gamma(\omega)$ is obvious, the introduction of the set $\bar{\Gamma}(\omega)$ is to control the effective interaction among the $A$-blocks after the decimation of the $B$-blocks (cf. [1]). 
In Appendix A we shall prove the following:

Lemma 2.2. Let conditions $\mathrm{C} 1, \mathrm{C} 2, \mathrm{C} 3$ be satisfied and $\varepsilon=\alpha-3 / 2>0$, then for all sufficiently large $q_{0}$ we have:

$$
\begin{aligned}
& \mathbb{P}\left\{\exists \alpha_{n-1}, \alpha_{n}:\left|\psi\left(\alpha_{n-1}, \alpha_{n}\right)\right|>\frac{1}{\left[q_{0}\left(\log _{+} n\right)^{\varrho / 1+\varepsilon}\right]^{\varepsilon / 4}}\right\} \\
& \leqq \\
& \left.\leqq \frac{1}{q_{0}(\log n)^{\varrho \frac{1+\varepsilon / 2}{1+\varepsilon}}}\right)^{(\log n)^{\frac{\varepsilon / 2}{1+\varepsilon}}} .
\end{aligned}
$$

Now for any given $\omega$ we are able to characterize the full set of the "bad" blocks:

$$
\tilde{\Gamma}(\omega)=\Gamma(\omega) \cup \bar{\Gamma}(\omega),
$$

and for $q_{0}$ sufficiently large, the following probability estimate holds (cf. Lemmas 2.1 and 2.2)

$$
\mathbb{P}\{Q(n) \in \tilde{\Gamma}(\omega)\} \leqq \exp \left\{-(\log n)^{\frac{\varepsilon / 2}{1+\varepsilon}}\right\} .
$$

Therefore if we take $\varrho \frac{\varepsilon / 2}{1+\varepsilon}>1$, then from Borel-Cantelli's lemma we conclude that $\tilde{\Gamma}(\omega)$ is bounded with probability one and we can define the set $C(\omega)$ as the minimal interval centered at the origin ending and starting with $B$-blocks containing $\tilde{\Gamma}(\omega)$.

It follows from the previous probability estimates that:

$$
\mathbb{P}\{\operatorname{diam} C(\omega) \geqq a\} \leqq \exp \left\{-(\log a)^{\frac{e}{1+2 / 2}}\right\},
$$

so that calling $\Omega_{0}$ the set of all realizations $\omega$ that give rise to finite $C$ 's, we have also

$$
\mathbb{P}\left(\Omega_{0}\right)=1 .
$$

In order to give the proof of Theorem 1 we will suppose for the sake of simplicity that the support of the cylindrical function is contained in $A(0)$, and we will start by considering the following expression:

where

$$
\langle f\rangle_{\omega, \beta, h}^{\Lambda}=\frac{\sum_{\sigma_{C}} f\left(\sigma_{C}\right) \exp \left\{-\beta H\left(\sigma_{C}\right)\right\} Z_{\Lambda \backslash C}^{\sigma_{C}}}{\sum_{\sigma_{C}} \exp \left\{-\beta H\left(\sigma_{C}\right)\right\} Z_{\Lambda \backslash C}^{\sigma_{C}}},
$$

and

$$
Z_{\Lambda \backslash C}^{\sigma_{C}} \equiv Z_{\Lambda \backslash C}^{\gamma_{C}}=\sum_{\alpha_{\Lambda \backslash C}} \exp \left\{-\beta H_{\Lambda \backslash C}\left(\sigma_{\Lambda \backslash C}\right)+W\left(\sigma_{C}, \sigma_{\Lambda \backslash C}\right)\right\},
$$

$$
C=\left\{\begin{array}{lll}
C(\omega) & \text { if } & C(\omega)=\bigcup_{-\left(2 n^{*}(\omega)-1\right)}^{+\left(2 n^{*}(\omega)-1\right)} Q(n) \\
B(0) \cup A(0) \cup B(1) & \text { if } & C(\omega)=\emptyset,
\end{array}\right.
$$

so that

$$
n^{*}=n^{*}(\omega)=\sup _{B(n) \subset C}|n| \quad \text { if } \quad C \neq \emptyset, \quad n^{*}=1 \quad \text { if } \quad C=\emptyset .
$$


Then we express $Z_{\Lambda \backslash C}^{\sigma C}$ using the notations introduced in Eq. (2.7)

$$
\begin{aligned}
Z_{\Lambda \backslash C}^{\sigma_{C}} \equiv & Z_{\Lambda \backslash C}^{\gamma_{C}}=\sum_{\alpha_{\Lambda \backslash C}} \exp \left\{\sum_{A(n) \in \Lambda \backslash C} a\left(\alpha_{n}\right)+\sum_{A(n), A(m) \in \Lambda \backslash C} W\left(\alpha_{n}, \alpha_{m}\right)\right\} \\
& \cdot \exp \left\{\sum_{\substack{A(n) \in \Lambda \backslash C \\
Q(m) \in C}} W\left(\alpha_{n}, \gamma_{m}\right)\right\} \sum_{\beta_{\Lambda \backslash C}}\left[\prod_{B(n) \in \Lambda \backslash C} \exp \left\{J\left(\beta_{n}, \alpha_{n-1}, \alpha_{n}\right)\right\}\right]\left[\prod_{L \in \mathscr{F}_{\Lambda, C}} \exp \left\{W_{L}\right\}\right],
\end{aligned}
$$

where $a\left(\alpha_{n}\right)$ is the self-interaction of the block $A(n), J\left(\beta_{n}, \alpha_{n-1}, \alpha_{n}\right)$ is defined as

$$
J\left(\beta_{n}, \alpha_{n-1}, \alpha_{n}\right)=a\left(\beta_{n}\right)+W\left(\beta_{n}, \alpha_{n-1}\right)+W\left(\beta_{n}, \alpha_{n}\right),
$$

and we have introduced the inverse temperature in all the interactions.

For every set $U: U \subset \mathbb{Z}, U \supset C, \mathscr{F}_{U, C}$ is the set of all pairs $L$ of blocks such that one of the elements of the pair is a $B$-block contained in $U$ and the other can be either a $B$-block in $U \backslash C$ or a nonadjacent $A$-block contained in $U \backslash C$ or, finally, a $Q$-block belonging to $C$. $W_{L}$ is the interaction energy among the two elements of $L$ (for the sake of simplicity we drop the explicit dependence of $W$ on the spin configurations).

Now we shall perform the sum over the $\beta$ variables in the right hand side of Eq. (2.20) (decimation procedure), and in complete analogy with [1], we shall obtain by means of a cluster expansion an explicit expression for the effective interaction among the $\alpha$ 's.

In this way we transform the original system into a physically equivalent system that, as we will see later, can be considered as in the small coupling (high temperature) region.

We define a polymer to be a collection $R=L_{1} \ldots L_{n}$ of bonds $L \in \mathscr{F}_{\mathbb{Z}, C}$ : for any pair $L_{i}, L_{j} \in R \exists L_{i_{1}} \ldots L_{i_{k}} \in R$ with $L_{i_{1}}=L_{i}$ and $L_{i_{k}}=L_{j}$ and such that $L_{i_{1}}$ and $L_{i_{n+1}}$ have a $B$-block in common.

Given $R$, we call $\tilde{R}$ the set of all $B$-blocks contained in $R$ and we call $\mathscr{R}_{U, C}$ the set of all polymers made only of bonds $\in \mathscr{F}_{U, C}$. We define the activity of the polymer as a function $\mathscr{H}: \mathscr{R}_{\mathbb{Z}, C} \rightarrow \mathbb{R}$ given by:

$$
\mathscr{H}(R)=\sum_{\beta_{\tilde{R}}} \prod_{B(n) \in \tilde{R}} \frac{\exp \left\{J\left(\beta_{n}, \alpha_{n-1}, \alpha_{n}\right)\right\}}{\sum_{\beta_{n}} \exp \left\{J\left(\beta_{n}, \alpha_{n-1}, \alpha_{n}\right\}\right.} \prod_{L \in R}\left(\exp \left\{W_{L}\right\}-1\right) .
$$

By standard manipulations we get

$$
\begin{aligned}
Z_{\Lambda \backslash C}^{\gamma_{C}=} & \sum_{\alpha_{\Lambda \backslash C}} \exp \left\{\sum_{A(n) \in \Lambda \backslash C} a\left(\alpha_{n}\right)+\sum_{A(n), A(m) \subset \Lambda \backslash C} W\left(\alpha_{n}, \alpha_{m}\right)+\sum_{\substack{A(n) \subset \Lambda \\
Q(m) \subset C}} W\left(\alpha_{n}, \gamma_{m}\right)\right\} \\
& \cdot \prod_{B(n) C \Lambda \backslash C} Z_{B(n)}^{\alpha_{n}-1, \alpha_{n} \Xi_{A, C}\left(\alpha_{\Lambda \backslash C}, \gamma_{C}\right)}
\end{aligned}
$$

where

$$
\Xi_{\Lambda, C}\left(\alpha_{\Lambda \backslash C}, \gamma_{C}\right)=1+\sum_{n \geqq 1} \sum_{\substack{R_{1} \ldots R_{n} \\ R_{i} \in \mathscr{R}_{A}, C \\ \tilde{R}_{i} \cap \tilde{R}_{j}=\emptyset}} \prod_{i=1}^{n} \mathscr{H}\left(R_{i}\right)
$$


The following lemma holds for $\Xi$ :

Lemma 2.3. If $q_{0}$ is large enough,

$$
\Xi_{\Lambda, C}\left(\alpha_{\Lambda \backslash C}, \gamma_{C}\right)=\exp \left[\sum_{n \geqq 1} \sum_{\substack{R_{1} \ldots R_{n} \\ R_{\imath} \in \mathscr{R}_{\Lambda, C}}} \varphi\left(R_{1} \ldots R_{n}\right) \prod_{i=1}^{n} \mathscr{H}\left(R_{i}\right)\right],
$$

with

$$
\varphi\left(R_{1} \ldots R_{n}\right)=\frac{1}{n !} \sum_{g \in \mathbb{G}_{n}\left(R_{1} \ldots R_{n}\right)}(-1)^{\#(\text { edges in } g)},
$$

where $\mathbb{G}_{n}\left(R_{1} \ldots R_{n}\right)$ is the set of connected graphs with $n$ vertices and edges $i, j$ corresponding to pairs $R_{i} R_{j}$ such that $\tilde{R}_{i} \cap \tilde{R}_{j}=\emptyset$. We set the sum equal to zero if $\mathbb{G}_{n}$ is empty and to 1 if $n=1$.

Proof. If $q_{0}$ is large enough, for any $r: 0<r<1$ we have

$$
|\mathscr{H}(R)| \leqq r^{|\tilde{R}|} \prod_{L \subset R} \frac{2\left\|W_{L}\right\|}{r^{2}},
$$

where we denote by $\|f\|$ the sup norm of the function $f$.

Using Lemma 2.1, we have

$$
\sup _{B(n) \in \mathbb{Z} \backslash C \backslash} \sum_{\substack{L \ni B(n) \\ L \subset \mathscr{F} \mathbb{Z} . C}} \frac{2}{r^{2}}\left\|W_{L}\right\| \leqq \frac{\text { Const }}{r^{2} q_{0}^{\lambda}}=\frac{k}{r^{2}} .
$$

If

$$
k \leqq \frac{r^{2}}{2} \log _{+}\left(\frac{1}{\sqrt{r}(2-\sqrt{r})}\right)
$$

Eq. (2.25) is obtained as a consequence of Lemma 2.1 of [1].

The result of the summation over the variables can then be written in the following way:

$$
\langle f\rangle_{\omega, \beta, h}^{\Lambda}=\frac{\sum_{\gamma_{C}} f\left(\gamma_{C}\right) \exp \left\{-\beta H\left(\gamma_{C}\right)\right\} \sum_{\alpha_{A} \backslash C} \exp \left\{-\bar{H}\left(\alpha_{\Lambda \backslash C}, \gamma_{C}\right)\right\}}{\sum_{\gamma_{C}} \exp \left\{-\beta H\left(\gamma_{C}\right)\right\} \sum_{\alpha_{\Lambda \backslash C} \backslash \bar{H}} \exp \left\{-\bar{H}\left(\alpha_{\Lambda \backslash C}, \gamma_{C}\right)\right\}},
$$

where

$$
\begin{aligned}
-\bar{H}\left(\alpha_{\Lambda \backslash C}, \gamma_{C}\right)= & \sum_{A(n) \subset \Lambda \backslash C} a\left(\alpha_{n}\right)+\sum_{A(n), A(m) \subset \Lambda \backslash C} W\left(\alpha_{n}, \alpha_{m}\right)+\sum_{\substack{A(n) \subset \Lambda \backslash C \\
Q(m) \subset C}} W\left(\alpha_{n}, \gamma_{m}\right) \\
& +\sum_{\substack{R_{1} \ldots R_{n} \\
R_{i} \in \mathscr{R}_{A}, C}} \varphi\left(R_{1} \ldots R_{n}\right) \prod_{i=1}^{n} \mathscr{H}\left(R_{i}\right) .
\end{aligned}
$$

Now to evaluate the sum over the $\alpha$ variables and get the final expression we shall perform a second polymer expansion. In order to define the polymers of the second kind, we extract suitable potentials from the renormalized hamiltonian. 
This particular way to collect the effective interactions among $\alpha$ 's will turn out to be convenient for further developments, even though at the moment it does not appear to be the most natural one.

For any given cluster of polymers $\underline{R}=R_{1} \ldots R_{n}$ we define the set

$$
E_{\underline{\underline{R}}}=\left[\bigcup_{i=1}^{n}\left(\bigcup_{L \subset R_{\mathrm{l}}} L\right)\right] \cup\left[\bigcup_{i=1}^{n} \bigcup_{B(n) \subset \tilde{R}}(A(n-1) \cup A(n))\right] .
$$

We call $\mathscr{E}_{U, C}$ the set of all possible $E_{R}$ with $R_{1} \ldots R_{n} \subset \mathscr{R}_{U, C}$.

For any given $E \in \mathscr{E}_{\mathbb{Z}, C}$ we can decompose it as $E=E_{A} \cup E_{B} \cup E_{C}$, where $E_{A}, E_{B}$, $E_{C}$ are respectively the set of $A$-blocks in $\mathbb{Z} \backslash C, B$-blocks in $\mathbb{Z} \backslash C, Q$-blocks in $C$ contained in $E$. Notice that $\forall E \in \mathscr{E}_{\mathbb{Z}, C}, E_{A} \neq \emptyset, E_{B} \neq \emptyset$.

We use the notation $\alpha_{E}=\alpha_{E_{A}}, \gamma_{E}=\gamma_{E_{C}}$. We define

$$
\tilde{V}_{E}\left(\alpha_{E}, \gamma_{E}\right)=\sum_{R_{1} \ldots R_{n}}^{E} \varphi\left(R_{1} \ldots R_{n}\right) \prod_{i=1}^{n} \mathscr{H}\left(R_{i}\right)
$$

where $\sum^{E}$ is over all $R: E_{\underline{R}}=E$, and write

$$
\sum_{R_{1} \ldots R_{n} \subset \mathscr{R}_{\Lambda, C}} \varphi\left(R_{1} \ldots R_{n}\right) \prod_{i=1}^{n} \mathscr{H}\left(R_{i}\right)=\sum_{E \in \mathscr{E}_{\Lambda}, C} \tilde{V}_{E}\left(\alpha_{E}, \gamma_{E}\right)
$$

Now we are able to express the renormalized Hamiltonian $\bar{H}$ appearing in the expression (2.29) as

$$
\begin{aligned}
-\bar{H}\left(\alpha_{\Lambda \backslash C}, \gamma_{C}\right)= & \sum_{A(n) \subset \Lambda \backslash C} \bar{a}\left(\alpha_{n}\right)+W\left(\alpha_{n^{*}}, \gamma_{2 n^{*}-1}\right) \\
& +W\left(\alpha_{-n^{*}}, \gamma_{-2 n^{*+1}}\right)+\sum_{D \subset A} V_{D}\left(\alpha_{D}, \gamma_{D}\right)
\end{aligned}
$$

where

$$
\bar{a}\left(\alpha_{n}\right)=a\left(\alpha_{n}\right)+\log \frac{Z_{B(n)}^{0, \alpha_{n}}}{Z_{B(n)}^{0,0}}+\log \frac{Z_{B(n+1)}^{\alpha_{n}, 0}}{Z_{B(n+1)}^{0,0}},
$$

and for any given set $D$ of $Q$-blocks the potentials $V_{D}$ are defined as follows:

$$
\begin{aligned}
& \text { for } D=A(n), Q(m): A(n) \in \mathbb{Z} \backslash C, Q(m) \in C, n, m \neq \pm n^{*}, \pm\left(2 n^{*}-1\right) V_{D}=W\left(\alpha_{n}, \gamma_{m}\right), \\
& \text { for } D=A(n), A(m) \in \mathbb{Z} \backslash C, n \neq m+1, V_{D}=W\left(\alpha_{n}, \alpha_{m}\right), \\
& \text { for } D=A(n), A(n+1) \in \mathbb{Z} \backslash C, V_{D}=W\left(\alpha_{n}, \alpha_{n+1}\right)+\psi\left(\alpha_{n}, \alpha_{n+1}\right)[\text { see Eq. (2.10)], } \\
& \text { for } D \equiv E \subset \mathscr{E}_{\mathbb{Z} . C}, V_{E}=\tilde{V}_{E}\left(\alpha_{E}, \gamma_{E}\right) \text {. }
\end{aligned}
$$

Any other $E$ is said to be nonadmissible and we put $V_{E} \equiv 0$. It is easy to realize that each term $V_{D}$ in Eq. (2.33) is "small."

Notice that:

a) We have included the two body term coming from the normalization of the first polymer expansion that is small for $A(n) \subset \Lambda \backslash C$.

b) We have not included in $V_{D}$ the interactions of the two blocks $A\left(n^{*}\right), A\left(-n^{*}\right)$ [see Eq. $\left(2.19^{\prime}\right)$ ] with $C$ because they can be large and then must be considered separately. 
The Second Polymer Expansion. A polymer $S$ is a collection of sets $D_{1} \ldots D_{n}$ of $Q$-blocks connected in the sense that $\forall D_{t}, D_{s} \in S \exists D_{i_{1}} \ldots D_{i_{r}}, D_{i_{1}}=D_{t}, D_{i_{r}}=D_{s}$ such that $D_{i_{h}}$ and $D_{i_{h+1}}$ have an $A$-block in common. Given a polymer $S=D_{1} \ldots D_{n}$ we will call $\tilde{S}$ the set of all the $A$-blocks belonging to $S$ and define its activity $\Theta(S)$ as :

$$
\Theta(S)=\sum_{\alpha_{\tilde{S}}} \prod_{A(n) \in \tilde{S}}\left(\frac{e^{\tilde{a}\left(\alpha_{n}\right)}}{\sum_{\alpha_{n}} e^{\tilde{a}\left(\alpha_{n}\right)}}\right) \prod_{D \in S}\left(e^{V_{D}\left(\alpha_{D}, \gamma_{D}\right)}-1\right),
$$

where

$$
\tilde{a}\left(\alpha_{n}\right)=\tilde{a}\left(\alpha_{n}\right), \quad \text { if }|n| \neq|n *|,
$$

$\tilde{a}\left(\alpha_{ \pm n^{*}}\right)=\bar{a}\left(\alpha_{ \pm n^{*}}\right)+W\left(\alpha_{ \pm n^{*}} \gamma_{ \pm\left(2 n^{*}-1\right)}\right)$.

Notice that if $S$ contains a nonadmissible $D$, then $\Theta(S)=0$.

Using the above definitions we easily get

$$
\langle f\rangle_{\omega, \beta, h}^{A}=\frac{\sum_{\gamma_{C}} f\left(\gamma_{C}\right) \exp \left\{-\beta H\left(\gamma_{C}\right)\right\} \sum_{\alpha_{n^{*}}} \exp \left\{\tilde{a}\left(\alpha_{n^{*}}, \gamma_{C}\right)+\tilde{a}\left(\alpha_{-n^{*}}, \gamma_{C}\right)\right\} \tilde{\Xi}_{\Lambda, C}\left(\gamma_{C}, \alpha_{ \pm n^{*}}\right)}{\sum_{\gamma_{C}} \exp \left\{-\beta H\left(\gamma_{C}\right)\right\} \sum_{\substack{\alpha_{n^{*}} \\ \alpha_{-n^{*}}}} \exp \left\{\tilde{a}\left(\alpha_{n^{*}}, \gamma_{C}\right)+\tilde{a}\left(\alpha_{-n^{*}}, \gamma_{C}\right)\right\} \tilde{\Xi}_{\Lambda, C}\left(\gamma_{C}, \alpha_{ \pm n^{*}}\right)}
$$

where

$$
\Xi_{\Lambda, C}\left(\gamma_{C}, \alpha_{ \pm n^{*}}\right)=1+\sum_{n \geqq 1} \sum_{\substack{S_{1} \ldots S_{n} \subset A \\ \tilde{S}_{i} \cap \tilde{\mathbf{S}}_{j}=\emptyset}} \prod_{i=1}^{n} \Theta\left(S_{i}\right)
$$

Lemma 2.4. If $q_{0}$ is sufficiently large:

$$
\tilde{\Xi}_{\Lambda, C}\left(\gamma_{C}, \alpha_{ \pm n^{*}}\right)=\exp \sum_{n \geqq 1} \sum_{S_{1} \ldots S_{n} \subset A} \varphi\left(S_{1} \ldots S_{n}\right) \prod_{i=1}^{n} \Theta\left(S_{i}\right) .
$$

Proof. For any $z: 0<z<1$ we can write, for $q_{0}$ sufficiently large:

$$
\Theta(S) \leqq z^{|\tilde{S}|} \prod_{D \subset S} \frac{2\left\|V_{D}\right\|}{z|D|_{Q}},
$$

where $|D|_{Q}$ means the number of $Q$-blocks in $D$. We have for $A(n) \in \mathbb{Z} \backslash C$,

$$
\begin{aligned}
\sum_{D \ni A(n)} \frac{2\left\|V_{D}\right\|}{z^{|D| Q}} \leqq & \sum_{\substack{Q(m): \\
2 m \pm 1 \neq n}} \frac{2}{z^{2}} \sup _{\alpha_{n} \gamma_{n}} \mid W\left(\alpha_{n}, \gamma_{m} \mid\right. \\
& +\frac{2}{z^{2}} \sup _{\alpha_{n-1}, \alpha_{n}, \alpha_{n+1}}\left(\left|\psi\left(\alpha_{n-1}, \alpha_{n}\right)\right|+\left|\psi\left(\alpha_{n}, \alpha_{n+1}\right)\right|\right) \\
& +\sum_{\substack{E \ni A(n) \\
E \in \mathscr{E} \mathbb{Z}, C}} \frac{2}{z^{|E|_{Q}}} \sum_{R_{1} \ldots R_{n} C A}^{E}\left|\varphi\left(R_{1} \ldots R_{n}\right)\right| \prod_{i=1}^{n}\left|\mathscr{H}\left(R_{i}\right)\right| \equiv \tilde{k}
\end{aligned}
$$

Now we can use the definition of $C(\omega)$ to treat the first two terms in the right hand side of Eq. (2.41) and standard methods of the theory of polymer expansion (see 
[8] and Eqs. (2.26) and (2.8) in [1] to treat the third one; so that we finally get for $q_{0}$ sufficiently large

$$
\tilde{k} \leqq \frac{1}{q_{0}^{\varepsilon / 4}}, \quad \text { and if } \quad \frac{1}{q_{0}^{\varepsilon / 4}} \leqq \frac{1}{2} \log _{+}\left(\frac{1}{\sqrt{z}(2-\sqrt{z})}\right),
$$

using again Lemma 2.1 of [1], Eq. (2.39) is proven.

Now we are able to rewrite Eq. (2.38) in the following way:

where

$$
\langle f\rangle_{\omega, \beta, h}^{\Lambda}=\frac{\sum_{\gamma_{C}, \alpha_{n^{*}, \alpha-n^{*}}} f\left(\gamma_{C}\right) \exp \left\{\tilde{H}\left(\gamma_{C}, \alpha_{ \pm n^{*}}\right)\right\}}{\sum_{\gamma_{C}, \alpha_{n^{*}, \alpha-n^{*}}} \exp \left\{\tilde{H}\left(\gamma_{C}, \alpha_{ \pm n^{*}}\right)\right\}}
$$

and

$$
\tilde{H}\left(\gamma_{C}, \alpha_{ \pm n^{*}}\right)=\hat{H}\left(\gamma_{C}, \alpha_{ \pm n^{*}}\right)+\sum_{\substack{S_{1} \ldots S_{n} \subset A \\\left(S_{1} \ldots S_{n}\right) \cap C \neq \emptyset}} \varphi\left(S_{1} \ldots S_{n}\right) \prod_{i=1}^{n} \Theta\left(S_{i}\right)
$$

$$
\hat{H}\left(\gamma_{C}, \alpha_{ \pm n^{*}}\right)=-\beta H\left(\gamma_{C}\right)+\tilde{a}\left(\alpha_{n^{*}}, \gamma_{C}\right)+\tilde{a}\left(\alpha_{-n^{*}}, \gamma_{C}\right)
$$

Notice that we have dropped the sum over the collection of polymers that do not intersect $C$ because they cancel out. This is the most important feature of Eq. (2.43) that we will use in the next section to prove Theorem 1.

\section{Proof of the Theorems}

We shall make use of the following lemmas proven in Appendix B.

Lemma 3.1. Consider the system of polymers described in the previous section, with activity given by Eqs. (2.35) and (2.36), then for any given integer $k$ there exists a constant $C_{k}$ such that for any finite set $T$ of $Q$-blocks we have:

$$
\left|D^{k} \sum_{\substack{S_{1} \ldots S_{n} \subset \mathbb{Z} \\\left(S_{1} \ldots S_{n}\right) \cap T \neq \emptyset}} \varphi\left(S_{1} \ldots S_{n}\right) \prod_{i=1}^{n} \Theta\left(S_{i}\right)\right| \leqq C_{k}\left(\log n_{T}\right)^{2 \varrho k}|T|_{Q},
$$

where $D^{k}$ is a derivative of order $k$ with respect to the variables $\beta$ and $h$ and $n_{T}$ $=\sup _{Q(n) \in T}|n|$.

Lemma 3.2. Under the same hypotheses of Lemma 3.1, consider two intervals $\Lambda, \Lambda^{\prime}$, $\Lambda^{\prime} \supset \Lambda \supset C$. Then for any $k$ there exists a constant $C_{k}$ such that $\forall$ set $T$ of $Q$-blocks contained in $\Lambda$, we have:

$$
\begin{aligned}
& D^{k}\left(\sum_{\substack{S_{1} \ldots S_{n} \subset A^{\prime} \\
\left(S_{1} \ldots S_{n}\right) \cap T \neq \emptyset}} \varphi\left(S_{1} \ldots S_{n}\right) \prod_{i=1}^{n} \Theta\left(S_{i}\right)-\sum_{\substack{S_{1} \ldots S_{n} \subset A \\
\left(S_{1} \ldots S_{n}\right) \cap T=\emptyset}} \varphi\left(S_{1} \ldots S_{n}\right) \prod_{i=1}^{n} \Theta\left(S_{i}\right)\right) \\
& \leqq \bar{c}_{k}\left(\ln n_{T}\right)^{2 \varrho k} \frac{|T|_{Q}}{d(T, \partial \Lambda)^{1 / 4}},
\end{aligned}
$$

where $d(T, \partial \Lambda)$ is the distance between $T$ and the boundary $\partial \Lambda$ of $\Lambda$. 
Now we are able to give an uniform bound to the $k$-derivative of any local quantity.

Proposition 3.1. Suppose conditions C1, C2, C3 be satisfied and $\frac{3}{2}<\alpha<2$, then $\forall$ integer $k, \exists$ a constant $\tilde{c}_{k}: \forall \omega \subset \Omega_{0}$ (cf. (2.15)) and $\forall$ cylindrical functions $f$ with support in the set of $Q$-blocks $T:$ :

$$
\left|D^{k}\langle f\rangle_{\omega, \beta, h}^{\Lambda}\right| \leqq \tilde{c}_{k}|C(\omega)|^{k+1}\left(\log n_{T}\right)^{2 \varrho k}\|f\|, \quad \forall \Lambda \supset C, T .
$$

Proof. Let us consider Eq. (2.43) and its generalization valid when the support of $f$ is not strictly contained in $C$ :

$$
\begin{aligned}
& \langle f\rangle_{\omega, \beta, h}^{\Lambda}
\end{aligned}
$$

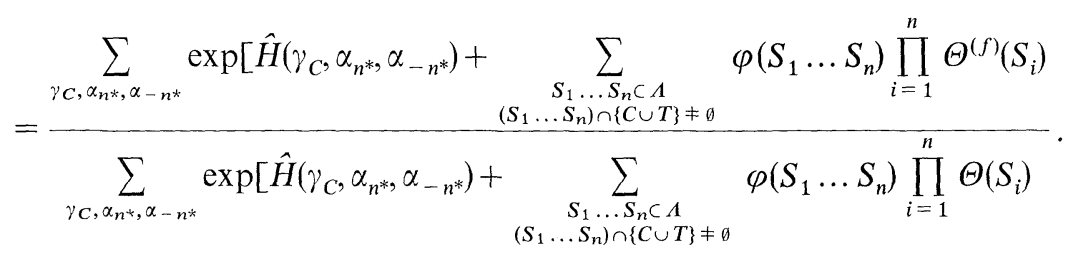

The activity $\Theta^{(f)}(S)$ is defined as in Eqs. (2.35) and (2.36), but starting from the modified Hamiltonian :

$$
-H^{(f)}\left(\sigma_{\Lambda}\right)=H\left(\sigma_{\Lambda}\right)+\log f\left(\sigma_{\Lambda}\right)
$$

where we have assumed, without any loss of generality, $f>0$ (if it is not so, we can add to $f$ a suitable constant). Then we write

$$
\langle f\rangle_{\omega, \beta, h}^{A}=\frac{\sum_{\gamma_{C}, \alpha_{n^{*}, \alpha_{-} n^{*}}} \exp \left[\tilde{H}\left(\gamma_{C}, \alpha_{ \pm n^{*}}\right)\right] G_{f}\left(\gamma_{C}, \alpha_{ \pm n^{*}}\right)}{\sum_{\gamma_{C}, \alpha_{n^{*}, \alpha_{-} n^{*}}} \exp \left[\tilde{H}\left(\gamma_{C}, \alpha_{ \pm n^{*}}\right)\right]},
$$

where $\tilde{H}$ is defined in Eq. (2.44) and $G_{f}\left(\gamma_{C}, \alpha_{ \pm n^{*}}\right)$ is given by:

$$
G_{f}\left(\gamma_{C}, \alpha_{ \pm n^{*}}\right)=\exp \left[\sum_{\substack{S_{1} \ldots S_{n} \subset A \\\left(S_{1} \ldots S_{n}\right) \cap\{T \cup C\} \neq \emptyset}} \varphi\left(S_{1} \ldots S_{n}\right)\left\{\prod_{i=1}^{n} \Theta^{(f)}\left(S_{i}\right)-\prod_{i=1}^{n} \Theta\left(S_{i}\right)\right\}\right] .
$$

If we consider a derivative of order $k$ we get a certain number of terms of the form:

$$
\prod_{i=1}^{s}\left\langle\left(\prod_{j=1}^{l_{l}} D_{j}^{h_{j}^{(2)}} \tilde{H}\right)\left(\prod_{j=1}^{m_{i}} D_{j}^{k_{j}^{(1)}} G_{f}\right)\right\rangle^{n_{i}}
$$

with

and

$$
\sum_{i=1}^{s} n_{i}\left(\sum_{j=1}^{l_{l}} h_{j}^{(i)}+\sum_{j=1}^{m_{l}} k_{j}^{(i)}\right)=k
$$

$$
\langle\cdot\rangle=\frac{\sum_{\gamma_{C}, \alpha_{ \pm n^{*}}} \cdot \exp \left[\tilde{H}\left(\gamma_{C}, \alpha_{ \pm n^{*}}\right)\right]}{\sum_{\gamma_{C}, \alpha_{ \pm n^{*}}} \exp \left[\tilde{H}\left(\gamma_{C}, \alpha_{ \pm n^{*}}\right)\right]} .
$$


We easily obtain

$$
\sup _{\gamma_{C}, \alpha_{ \pm n^{*}}}\left|D^{k} \hat{H}\left(\gamma_{C}, \alpha_{ \pm n^{*}}\right)\right| \leqq \hat{C}_{k}|C|^{k}
$$

for some constant $\hat{C}_{k}$. Note that Lemma 3.1 generalizes to the case of $\Theta$ replaced by $\Theta^{(f)}$. Applying this concludes the proof.

Proof of Theorem 1. We shall show that $\forall k$, given a cylindrical function $f$ :

$$
\left|D^{k}\left(\langle f\rangle_{\omega, \beta, h}^{\Lambda^{\prime}}-\langle f\rangle_{\omega, \beta, h}^{\Lambda}\right)\right|_{\underset{\Lambda / \mathbb{Z}}{\longrightarrow}} 0, \quad \forall \omega \in \Omega_{0},
$$

uniformly for $\beta$ and $h$ varying in finite intervals and uniformly in $\Lambda^{\prime} \supset \Lambda$. Starting from Eq. (3.4), we can write

$$
\begin{aligned}
& \langle f\rangle_{\omega, \beta, h}^{\Lambda^{\prime}}-\langle f\rangle_{\omega, \beta, h}^{\Lambda} \\
& \quad=\left\langle f\left(\exp \left[\tau_{f}\right]-1\right)\right\rangle_{\omega, \beta, h}^{\Lambda}-\langle f\rangle_{\omega, \beta, h}^{\Lambda^{\prime}}\langle\exp [\tau]-1\rangle_{\omega, \beta, h}^{\Lambda},
\end{aligned}
$$

where

$$
\begin{gathered}
\tau=\sum_{\substack{S_{1} \ldots S_{n} \subset \Lambda^{\prime} \backslash A \\
\left(S_{1} \ldots S_{n}\right) \cap(C \cup T) \neq \emptyset}} \varphi\left(S_{1} \ldots S_{n}\right) \prod_{i=1}^{n} \Theta\left(S_{i}\right), \\
\tau_{f}=\sum_{\substack{S_{1} \ldots S_{n} \subset A^{\prime} \backslash \Lambda \\
\left(S_{1} \ldots S_{n}\right) \cap(C \cup T) \neq \emptyset}} \varphi\left(S_{1} \ldots S_{n}\right) \prod_{i=1}^{n} \Theta^{(f)}\left(S_{i}\right), \\
\sum_{i=1}
\end{gathered}
$$

and $T$ is the support of $f$. Then from Lemma 3.2 and from Proposition 3.1, we have

$$
\left|D^{k}\left(\langle f\rangle_{\omega, \beta, h}^{\Lambda^{\prime}}-\langle f\rangle_{\omega, \beta, h}^{\Lambda}\right)\right| \leqq \bar{c}_{k}\left(\log n_{T}\right)^{2 \varrho k}|T|_{Q} \frac{|C(\omega)|^{k+1}}{d(T, \partial \Lambda)^{1 / 4}}
$$

This proves (3.9) and concludes the proof of the first part of Theorem 1.

To prove the second part, it is sufficient to remark that the uniform bound (3.3) is $L^{1}(\mathbb{P}(d \omega))$ as a consequence of the probability estimate given by Eq. (2.15); then the result follows from the dominated convergence theorem.

Proof of Theorem 2. With the hypotheses of Theorem 2 the following results proved by Khanin and Sinai in [2] hold in our case:

$$
\exists \lim _{\Lambda \ngtr \mathbb{Z}} \mathbb{E}\left(F_{\Lambda}(\omega, \beta, h)\right)=F(\beta, h),
$$

$$
\lim _{\Lambda>\mathbb{Z}} F_{\Lambda}(\omega, \beta, h)=F(\beta, h) . \mathbb{P} \text {-a.e. }
$$

Now for every finite interval $\Lambda$, we can write

$$
\begin{aligned}
& \frac{d}{d h} F_{\Lambda}(\omega, \beta, h)=\frac{1}{|\Lambda|} \sum_{t \in \Lambda}\left\langle\sigma_{t}\right\rangle_{\omega, \beta, h}^{\Lambda}, \\
& \frac{d}{d \beta} F_{\Lambda}(\omega, \beta, h)=\frac{1}{|\Lambda|} \sum_{\substack{t_{1}, t_{2} \in \Lambda \\
t_{1} \neq t_{2}}} \varepsilon_{t_{1} t_{2}} \frac{\left\langle\sigma_{t_{1}} \sigma_{t_{2}}\right\rangle_{\omega, \beta, h}^{A}}{\left|t_{1}-t_{2}\right|^{\alpha}} .
\end{aligned}
$$


Then to prove the theorem it is sufficient to show that $\forall k>0, \forall \omega \in \Omega_{0}$ and uniformly for $\beta$ and $h$ varying in finite intervals :

$$
\begin{gathered}
\exists \lim _{\Lambda>\mathbb{Z}} D^{k} \frac{1}{|\Lambda|} \sum_{t \in \Lambda}\left\langle\sigma_{t}\right\rangle_{\omega, \beta, h}^{\Lambda}=\lim _{\Lambda>\mathbb{Z}} \mathbb{E}\left(D^{k} \frac{1}{|\Lambda|} \sum_{t \in \Lambda}\left\langle\sigma_{t}\right\rangle_{\omega, \beta, h}^{\Lambda}\right), \\
\exists \lim _{\Lambda>\mathbb{Z}} D^{k} \frac{1}{|\Lambda|} \sum_{\substack{t_{1}, t_{2} \in \Lambda \\
t_{1} \neq t_{2}}} \varepsilon_{t_{1} t_{2}} \frac{\left\langle\sigma_{t_{1}} \sigma_{t_{2}}\right\rangle_{\omega, \beta, h}^{\Lambda}}{\left|t_{1}-t_{2}\right|^{\alpha}}=\lim _{\Lambda>\mathbb{Z}} \mathbb{E}\left(D^{k} \frac{1}{|\Lambda|} \sum_{\substack{t_{1}, t_{2} \in \mathbb{Z} \\
t_{1} \neq t_{2}}} \varepsilon_{t_{1} t_{2}} \frac{\left\langle\sigma_{t_{1}} \sigma_{t_{2}}\right\rangle_{\omega, \beta, h}^{\Lambda}}{\left|t_{1}-t_{2}\right|^{\alpha}}\right) .
\end{gathered}
$$

To get this strong law of large numbers we introduce a family of overlapping volumes $V_{t}$ centered at each site $t$, whose size is increasing as a suitable power law of the distance of the site $t$ from the origin.

Let, $\forall t \in \mathbb{Z}$

$$
V_{t}=A\left(n_{t}-\left[t^{1 / 4}\right]\right) \cup\left(\bigcup_{n=n_{t}-\left[t^{1 / 4}\right]+1}^{n_{t}+\left[t^{1 / 4}\right]} B(n) \cup A(n)\right),
$$

where $n_{t}$ is the index of the $B$ or $A$ block to which the lattice site belongs. It is easy to see that $\left|V_{t}\right| \sim$ const $q_{0} t^{1 / 4}\left(\log _{+} t\right)^{\varrho}$. We can write (dropping for simplicity the dependence on $\omega, \beta, h)$ :

$$
\begin{aligned}
\frac{1}{|\Lambda|} \sum_{t \in \Lambda} D^{k}\left\langle\sigma_{t}\right\rangle= & \frac{1}{|\Lambda|} \sum_{t \in \Delta \Lambda} D^{k}\left\langle\sigma_{t}\right\rangle^{\Lambda} \\
& +\frac{1}{|\Lambda|} \sum_{t \in \Lambda \backslash \Delta \Lambda} D^{k}\left\langle\sigma_{t}\right\rangle^{V_{t}}+\frac{1}{|\Lambda|} \sum_{t \in \Lambda \backslash \Delta \Lambda} D^{k}\left(\left\langle\sigma_{t}\right\rangle^{\Lambda}-\left\langle\sigma_{t}\right\rangle^{V_{t}}\right),
\end{aligned}
$$

where

$$
\Delta \Lambda=\left\{t: \frac{1}{2}|\Lambda|-\left[\sqrt{\frac{|\Lambda|}{2}}\right] \leqq t \leqq \frac{1}{2}|\Lambda|\right\}, \quad \text { for } \quad \Lambda=\left(-\frac{|\Lambda|}{2}, \frac{|\Lambda|}{2}\right)
$$

For each $t \in \Lambda \backslash \Delta \Lambda$, we have that $V_{t}$ is strictly contained in $\Lambda$ if $\Lambda$ is large enough.

The first sum in Eq. (3.17) can be estimated using Proposition 3.1 as:

$$
\frac{1}{|\Lambda|} \sum_{t \in \Delta \Lambda}\left|D^{k}\left\langle\sigma_{t}\right\rangle^{\Lambda}\right| \leqq \tilde{C}_{k}|C(\omega)|^{k+1}(\log |\Lambda|)^{2 \varrho k} \frac{\sqrt{|\Lambda|}}{|\Lambda|}
$$

The terms of the third sum are estimated using Eq. (3.12)

$$
\left|D^{k}\left(\left\langle\sigma_{t}\right\rangle^{\Lambda}-\left\langle\sigma_{t}\right\rangle^{V}\right)\right| \leqq \overline{\bar{C}}_{k}\left(\log n_{t}\right)^{2 \varrho k}|C(\omega)|^{k+1} \frac{1}{t^{1 / 16}}
$$

The problem of the existence of the limit of $\frac{1}{|\Lambda|} \sum_{i \in \Lambda} D^{k}\left\langle\sigma_{i}\right\rangle^{\Lambda}$ is then reduced to the existence of the limit of $\frac{1}{|\Lambda|} \sum_{t \in \Lambda} D^{k}\left\langle\sigma_{t}\right\rangle^{V_{t}}$.

Set :

$$
\xi_{t}=D^{k}\left\langle\sigma_{t}\right\rangle^{V_{t}}-\mathbb{E}\left(D^{k}\left\langle\sigma_{t}\right\rangle^{V_{t}}\right.
$$


The following proposition is true:

\section{Proposition 3.2.}

$$
\lim _{\Lambda \wedge \mathbb{Z}} \frac{1}{|\Lambda|} \sum_{t \in \Lambda} \xi_{t}=0, \quad \mathbb{P} \text {-a.e. }
$$

Proof. Since $\xi_{t_{1}}$ and $\xi_{t_{2}}$ are independent if $t_{1}$ and $t_{2}$ are sufficiently far apart, we have for $\Lambda$ sufficiently large

$$
\mathbb{E}\left(\left(\frac{1}{|\Lambda|} \sum_{t \in \Lambda} \xi_{t}\right)^{4}\right) \leqq \frac{\text { const }}{|\Lambda|^{4}} \Lambda^{2+2 \cdot 1 / 4}(\ln \Lambda)^{2 \varrho k+1} \leqq \frac{1}{|\Lambda|^{4 / 3}},
$$

so that using Chebichev's inequality we get

$$
\sum_{|\Lambda|=1}^{\infty} \mathbb{P}\left(\frac{1}{|\Lambda|}\left|\sum_{t \in \Lambda} \xi_{t}\right|>\varepsilon\right) \leqq \frac{1}{\varepsilon^{4}} \sum_{|\Lambda|=1}^{\infty} \frac{1}{|\Lambda|^{4 / 3}}<\infty .
$$

Now using Theorem 1 and condition C4, it is easy to show that

$$
\begin{aligned}
\lim _{\Lambda>\mathbb{Z}} & \frac{1}{|\Lambda|} \sum_{t \in \Lambda} \mathbb{E}\left(D^{k}\left\langle\sigma_{t}\right\rangle^{V_{t}}\right) \\
= & \lim _{\Lambda>\mathbb{Z}} \frac{1}{|\Lambda|} \sum_{t \in \Lambda} \mathbb{E}\left(D^{k}\left\langle\sigma_{t}\right\rangle^{\Lambda}\right)=\lim _{\Lambda>\mathbb{Z}} \mathbb{E}\left(D^{k}\left\langle\sigma_{0}\right\rangle^{\Lambda}\right) .
\end{aligned}
$$

It is easily seen that the same kind of arguments apply to the case :

$$
\frac{1}{|\Lambda|} \sum_{\substack{t_{1}, t_{2} \in \Lambda \\ t_{1} \neq t_{2}}} \frac{\varepsilon_{t_{1} t_{2}}}{\left|t_{1}-t_{2}\right|^{\alpha}} D^{k}\left\langle\sigma_{t_{1}} \sigma_{t_{2}}\right\rangle^{\Lambda}
$$

In fact the denominator $\left|t_{1}-t_{2}\right|^{\alpha}$ controls the behaviour at large distances and for small $\left|t_{1}-t_{2}\right|$ we are back to the previous situation. This ends the proof of Theorem 2.

\section{Remarks}

Remark 1. In the sequel we will discuss a one-dimensional model where it is possible to rigorously prove the existence of a Griffiths singularity $[4,5]$. We will actually show that for our model $\exists \beta_{C}: \forall \beta>\beta_{C}$, the average magnetization is nonanalytic in $h$ at $h=0$, meanwhile it is still $C^{\infty}$ at every $\beta$ and $h$.

Let us consider a system described by the Hamiltonian (1.1) where the $\varepsilon_{t t^{\prime}}$ are allowed to take the values 0 and 1 with the following probability distribution

$$
\begin{aligned}
& \mathbb{P}\left(\varepsilon_{t t^{\prime}}=1\right)=f\left(\left|t-t^{\prime}\right|\right) \leqq \frac{p}{\left|t-t^{\prime}\right|^{1+\delta}}, \quad \delta>0, \\
& \mathbb{P}\left(\varepsilon_{t t^{\prime}}=0\right)=1-f\left(\left|t-t^{\prime}\right|\right) .
\end{aligned}
$$

We call $\mathscr{L}$ the set of all the pairs (bonds) $b=\left(t, t^{\prime}\right), t, t^{\prime} \in \mathbb{Z}$; the space of the realizations of our random field is : $\Omega=(0,1)^{\mathscr{L}}$. We can represent $\Omega$ as the set $\mathscr{S}$ of all subsets $S$ of $\mathscr{L}: S$ being the set of bonds $b$ with $\varepsilon_{b}=1$. 
Given an $S \subset \mathscr{S}$ we can decompose it into its maximal connected components called clusters. A cluster $\Gamma$ is a (generally infinite) set of bonds $\in \mathscr{L}$ connected in the sense that for any pair of bonds $b_{i}, b_{j} \in \Gamma, \exists b_{i_{1}} \ldots b_{i_{r}} \in \Gamma$, with $b_{i_{r}}=b_{j}, b_{i_{1}}=b_{i}$, $b_{i_{k}} \cap b_{i_{k+1}} \neq \emptyset, k=1, \ldots, r-1$ and maximal with respect to this connection. We want to show that, for $p$ in Eq. (4.1) sufficiently small, the probability of percolation (i.e. the probability that the origin belongs to an infinite cluster) is zero.

For any finite interval $\Lambda$ centered at the origin, consider the following event $E_{O, \Lambda}$ : the origin belongs to a cluster intersecting $\mathbb{Z} \Lambda \equiv \Lambda^{C}$. The following inequality holds :

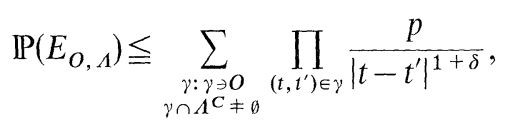

where $\gamma$ is any finite set of connected bonds in $\mathbb{Z}$. We have:

$$
\mathbb{P}\left(E_{O, \Lambda}\right) \leqq \frac{\text { const }}{|\Lambda|^{\delta / 2}} \sum_{\gamma \ni O} \xi(\gamma)
$$

with

$$
\xi(\gamma)=\prod_{\left(t, t^{\prime}\right) \in \gamma} \frac{p}{\left|t-t^{\prime}\right|^{1+\delta / 2}}
$$

To estimate the sum on the right hand side of Eq. (4.3) one can use arguments typical of the high temperature expansions. For instance, using the method developed in Appendix A of [1], one can easily see that the "activity $\xi(\gamma)$ " of the "polymer" $\gamma$ is so small that

$$
\sum_{\gamma \ni O} \xi(\gamma)<+\infty
$$

Than from Eq. (4.3), since $\mathbb{P}\left(E_{O, \Lambda}\right) \underset{\Lambda \times \mathbb{Z}}{\longrightarrow} 0$, we conclude the proof of the absence of percolation.

Now, following Griffiths, we are able to write the average (quenched) magnetization in the thermodynamic limit as

$$
\begin{aligned}
\lim _{\Lambda \ngtr \mathbb{Z}} \int \mathbb{P}(\mathrm{d} \omega)\left\langle\sigma_{O}\right\rangle_{\omega, \beta, h}^{\Lambda} & =\sum_{\Gamma \ni O} \mathbb{P}(\Gamma) \frac{1}{|\tilde{\Gamma}|} \sum_{t \in \tilde{\Gamma}}\left\langle\sigma_{t}\right\rangle_{\Gamma, \beta, h} \\
& \equiv m(\beta, h),
\end{aligned}
$$

where the sum is over all finite clusters $\Gamma$ passing through the origin [the $\mathbb{P}(\Gamma)$ 's are the probabilities of their occurrence], $\tilde{\Gamma}$ is the set of all sites $t$ belonging to bonds in $\Gamma$, and $\left\langle\sigma_{t}\right\rangle_{\Gamma}$ is the magnetization in the site $t$ evaluated in the finite volume $\tilde{\Gamma}$ with zero boundary conditions when only the set of bonds belonging to $\Gamma$ is present. We further notice that for every finite interval $\Lambda$ the cluster $\Gamma_{\Lambda}$ obtained by putting $\varepsilon_{t t^{\prime}}$ $=1$ for any $t, t^{\prime} \in \Lambda$ and $\varepsilon_{t t^{\prime}}=0 \forall t \in \Lambda, t^{\prime} \in \mathbb{Z} \backslash \Lambda$ has nonzero probability. In fact we have:

$$
\mathbb{P}\left(\Gamma_{\Lambda}\right) \geqq\left(\frac{p}{|\Lambda|^{1+\delta}}\right)^{|\Lambda|^{2}}\left[\prod_{t \in \mathbb{Z}}\left(1-\frac{p}{|t|^{1+\delta}}\right)\right]^{|\Lambda|}>0
$$


We see that we are exactly in the situation discussed by Griffiths in [5] for a dilute 2-dimensional Ising model, so that we can conclude that for $\forall \alpha<2, \forall \beta$ $>\beta_{C}(\alpha)$, where $\beta_{C}(\alpha)$ is the inverse critical temperature of the fully ferromagnetic system (cf. [9]), the quenched magnetization is nonanalytic in $h$ at $h=0$.

As far as the $C^{\infty}$ properties are concerned, let us consider, as an example, $\frac{\partial^{k}}{\partial h^{k}} m(\beta, h)$. It is easy to convince ourselves that:

$$
\begin{aligned}
\sum_{\Gamma \ni O} \mathbb{P}(\Gamma) \frac{1}{|\tilde{\Gamma}|} \sum_{t \in \tilde{\Gamma}} \frac{\partial^{k}}{\partial h^{k}}\left\langle\sigma_{t}\right\rangle_{\Gamma, \beta, h} & \leqq C_{k} \sum_{\Gamma \ni O} \mathbb{P}(\Gamma)|\tilde{\Gamma}|^{k} \\
& \leqq C_{k} \sum_{n=1}^{\infty} n^{k}(\sqrt{p})^{n-1} \cdot \sum_{\gamma \ni O} \tilde{\xi}(\gamma)<+\infty
\end{aligned}
$$

for

$$
\tilde{\xi}(\gamma)=\prod_{\left(t, t^{\prime}\right) \in \gamma}\left(\frac{\sqrt{p}}{\left|t-t^{\prime}\right|^{1+\delta}}\right)
$$

and $p$ sufficiently small (the constant $C_{k}$ depends only on $k$ ); so that the series that formally expresses $\frac{\partial^{k}}{\partial h^{k}} m(\beta, h)$ is uniformly convergent in $h$.

Remark 2. In the previous sections we have used "zero boundary conditions" [cf. Eqs. (1.1) and (1.2)], but it is easy to verify that, with minor changes, it is possible to prove exactly the same results in the general case: i.e. all the limits quoted in Theorems 1 and 2 exist and are independent of the boundary conditions.

Remark 3. In [1] the problem of the analyticity for a one dimensional system with translationally invariant finite first moment interactions was considered. The hypothesis of translation invariance was introduced to be able to apply Ruelle's theory [10] to the term $\psi\left(\alpha_{i}, \alpha_{i+1}\right)$ of the effective Hamiltonian [cf. Eq. (2.10)]. In the present paper, to prove our theorems we have to consider nontranslationally invariant potentials; in particular to treat the term $\psi\left(\alpha_{i}, \alpha_{i+1}\right)$ we use an approach originally due to Dobrushin [11] that applies also to nontranslationally invariant systems. The following theorem that generalizes the main result given in [1] easily follows as an adaptation of the method used in the present paper.

Theorem 3. Consider the Banach space $\mathscr{E}$ of the complex-valued functions on the finite subsets of $\mathbb{Z}$ such that:

$$
\||\Phi|\|=\sum_{X \ni O} \frac{\operatorname{diam} X+1}{|X|} \sup _{t \in \mathbb{Z}}|\Phi(\mathrm{X}+\mathrm{t})|<+\infty .
$$

For $\Phi_{0}, \Psi_{1} \ldots \Psi_{d} \in \mathscr{E}^{\prime},\left\|\left|\Psi_{1}\right|\right\|=1$, consider the free energy

$$
F_{\Lambda}\left(\Phi+\sum_{i=1}^{d} \lambda_{i} \Psi_{i}\right)=\frac{1}{|\Lambda|} \log \sum_{X_{A}} \exp \left(-H_{\Lambda}^{i}\left(X_{\Lambda}\right)\right)
$$


where $\underline{\lambda}=\left(\lambda_{1}, \ldots, \lambda_{d}\right)$

$$
\begin{gathered}
H_{\Lambda}^{\underline{\lambda}}\left(X_{\Lambda}\right)=\sum_{X \subset \Lambda} \Phi^{\underline{\lambda}}(X) \prod_{t \in X} x_{t}, \\
\Phi^{\underline{\lambda}}=\Phi_{0}+\sum_{i=1}^{d} \lambda_{i} \Psi_{i} \quad x_{t} \in\{0,1\},
\end{gathered}
$$

and the expectation of any cylindrical function $f$ :

$$
\langle f\rangle \frac{\lambda}{\Lambda}=\sum_{X_{\Lambda}} \frac{f\left(X_{\Lambda}\right) \exp \left[-H_{\Lambda}^{\lambda}\left(X_{\Lambda}\right)\right]}{\sum_{X_{\Lambda}} \exp \left[-H_{\bar{\Lambda}}^{\lambda}\left(X_{\Lambda}\right)\right]}
$$

then $\exists$ a sphere $\omega$ in $\mathbb{C}^{d}$ such that:

$$
\exists \lim _{\Lambda \supset \mathbb{Z}}\langle f\rangle \frac{\lambda}{\Lambda}
$$

and defines a holomorphic function of $\underline{\lambda}$ in $\omega$.

2) If the limit

$$
\lim _{\Lambda \ngtr \mathbb{Z}} F_{\Lambda}\left(\Phi_{0}\right)
$$

exists, then

$$
F\left(\Phi_{0}+\sum \lambda_{i} \Psi_{i}\right)=\lim _{\Lambda>\mathbb{Z}} F_{\Lambda}\left(\Phi_{0}+\sum \lambda_{i} \Psi_{i}\right)
$$

exists and is holomorphic in $\underline{\lambda}$ on $\omega$.

\section{Appendix A}

Proof of Lemma 2.2. We start by proving an auxiliary lemma:

Lemma A.1. Let $V$ be a volume partitioned in $\left[n^{u}\right]$ volumes $V_{i}$ each of length $n$ (i.e. $\left|V_{i}\right|=n$ and $\left.|V|=n^{1+u}\right)$, and let $\sigma_{+}\left(\sigma_{-}\right)$be the configuration of the spins in a finite volume on the right (on the left) of the volume $V$. If $n$ is large enough, $u \leqq 1$ and $\varepsilon=\alpha$ $-\frac{3}{2}$. Then the following probability estimate holds:

$$
\mathbb{P}\left\{\forall \sigma_{+}, \sigma_{-}\left|\frac{Z_{V}^{\sigma_{-}, \sigma_{+}} Z_{V}^{0,0}}{Z_{V}^{\sigma_{-}, 0} Z_{V}^{0, \sigma_{+}}}-1\right|<\frac{1}{|V|^{\varepsilon / 4}}\right\}>1-\frac{1}{|V|^{1 / 2}} .
$$

Proof. If we call $W\left(\sigma_{V}, \sigma_{+}\right)\left(W\left(\sigma_{V}, \sigma_{-}\right)\right)$the interaction energy of the volume $V$ with the right (left) boundary and $\langle\cdot\rangle_{0}$ the expectation with the probability measure

$$
q_{V}\left(\sigma_{V}\right)=\frac{\exp \left\{-\beta H_{V}\left(\sigma_{V}\right)\right\}}{\sum_{\sigma_{V}} \exp \left\{-\beta H_{V}\left(\sigma_{V}\right)\right\}},
$$

we have

$$
\frac{Z_{V}^{\sigma_{+}, \sigma_{-}} Z_{V}^{0,0}}{Z_{V}^{\sigma_{+}, 0} Z_{V}^{0, \sigma_{-}}}=\frac{\left\langle\exp \left\{W\left(\sigma_{V}, \sigma_{-}\right)\right\} \exp \left\{W\left(\sigma_{V}, \sigma_{-}\right)\right\}\right\rangle_{0}}{\left\langle\exp \left\{W\left(\sigma_{V}, \sigma_{-}\right)\right\}\right\rangle_{0}\left\langle\exp \left\{W\left(\sigma_{V}, \sigma_{+}\right)\right\}\right\rangle_{0}}
$$


Recalling that $V=\bigcup_{0}^{N} V_{i}$ with $N=n^{u}$ and setting:

$$
\begin{aligned}
& f_{1}=f_{1}\left(\sigma_{V_{0}}\right)=\exp \left\{W\left(\sigma_{V_{0}}, \sigma_{-}\right)\right\} \\
& f_{2}=f_{2}\left(\sigma_{V_{N}}\right)=\exp \left\{W\left(\sigma_{V_{N}}, \sigma_{+}\right)\right\}
\end{aligned}
$$

By Lemma 3 of [3] we have with probability larger than $1-\exp \left\{-n^{1 / 2(1+\delta / 2)}\right\}$

$$
\begin{aligned}
\frac{\left\langle f_{1} f_{2}\right\rangle_{0}}{\left\langle f_{1}\right\rangle_{0}\left\langle f_{2}\right\rangle_{0}} \exp \left\{-\frac{\text { const }}{n^{\varepsilon-\delta}}\right\} & \leqq \frac{\left\langle\exp \left\{W\left(\sigma_{V}, \sigma_{-}\right)\right\} \exp \left\{W\left(\sigma_{V}, \sigma_{+}\right)\right\}\right\rangle_{0}}{\left\langle\exp \left\{W\left(\sigma_{V}, \sigma_{-}\right)\right\}\right\rangle_{0}\left\langle\exp \left\{W\left(\sigma_{V}, \sigma_{-}\right)\right\}\right\rangle_{0}} \\
& \leqq \frac{\left\langle f_{1} f_{2}\right\rangle_{0}}{\left\langle f_{1}\right\rangle_{0}\left\langle f_{2}\right\rangle_{0}} \exp \left\{\frac{\text { const }}{n^{\varepsilon-\delta}}\right\}
\end{aligned}
$$

for any $n$ sufficiently large and $\delta: 0<\delta<\frac{1}{2}$. But

$$
\begin{aligned}
\left\langle f_{1} f_{2}\right\rangle_{0} & =\sum_{\sigma_{V}} q_{V}\left(\sigma_{V}\right) f_{1}\left(\sigma_{V_{0}}\right) f_{2}\left(\sigma_{V_{N}}\right) \\
& =\sum_{\sigma_{V_{0}}} q_{V_{0}, V}\left(\sigma_{V_{0}}\right) f_{1}\left(\sigma_{V_{0}}\right) \sum_{\sigma_{V_{N}}} q_{V_{N}, V \backslash V_{0}, \sigma_{V_{0}}}\left(\sigma_{V_{N}}\right) f_{2}\left(\sigma_{V_{N}}\right),
\end{aligned}
$$

where $q_{V_{0}, V}(\cdot)$ is the relativization of $q_{V}(\cdot)$ to $\sigma_{V_{0}}$, and $q_{V_{N}, V \backslash V_{0}, \sigma_{V_{0}}}(\cdot)$ is the relativization to $\sigma_{V_{N}}$ of the conditional probability on the values $\sigma_{V \backslash V_{0}}$ evaluated by means of $q_{V}(\cdot)$ with conditions $\sigma_{V_{0}}$. Then

$$
\begin{aligned}
\left\langle f_{1} f_{2}\right\rangle_{0}- & \left\langle f_{1}\right\rangle_{0}\left\langle f_{2}\right\rangle_{0} \mid \leqq \sum_{\sigma_{V_{0}}} q_{V_{0}, V}\left(\sigma_{V_{0}}\right) f_{1}\left(\sigma_{V_{0}}\right) \\
& \cdot\left[\sum_{\sigma_{V_{N}}}\left|q_{V_{N}, V \mid V_{0}, \sigma_{V_{0}}}\left(\sigma_{V_{N}}\right)-\sum_{\sigma_{V_{0}^{\prime}}} q_{V_{N}, V \mid V_{0}, \sigma_{V_{0}}}\left(\sigma_{V_{N}}\right) q_{V}\left(\sigma_{V_{0}}^{\prime}\right)\right| f_{2}\left(\sigma_{V_{N}}\right)\right] \\
\leqq & 2 \max _{\sigma_{V_{0}}, \sigma_{V_{N}}}\left|f_{1} f_{2}\right| \sup _{\sigma_{V_{0}}^{\prime}, \sigma_{V_{0}}} \varrho\left(q_{V_{N}, V \mid V_{0}, \sigma_{V_{0}}}(\cdot), q_{V_{N}, V \mid V_{0}, \sigma_{V_{0}}}(\cdot)\right),
\end{aligned}
$$

where $\varrho(\cdot, \cdot)$ is the distance in variation and we have used the following inequality: Let $X$ be a finite space, $p(x), p_{i}(x) i=1,2, ., n$, probability measures on $X, \lambda_{i}>0$ $(i=1,2, \ldots, n): \sum_{0}^{n} \lambda_{i}=1$, then :

$$
\begin{aligned}
\sum_{x \in X}\left|p(x)-\sum_{j=1}^{n} \lambda_{j} p_{j}(x)\right| & \leqq \sum_{x \in X} \sum_{j=1}^{n} \lambda_{j}\left|p(x)-p_{j}(x)\right| \\
& \leqq \max _{j} \varrho\left(p(\cdot), p_{j}(\cdot)\right) .
\end{aligned}
$$

In [3] it is shown that, with probability larger than:

$$
\begin{aligned}
1- & \operatorname{const} n^{u} \exp \left\{-p(n)^{1 / 2(1+\delta / 2)}\right\}-\exp \left\{-n^{1 / 2(1+\delta / 2)}\right\} \\
& \cdot \sup _{\sigma_{V_{0}}, \sigma_{V_{0}}} \varrho\left(q_{V_{N}, V \mid V_{0}, \sigma_{V_{0}}}(\cdot), q_{V_{N}, V \mid V_{0}, \sigma^{\prime} V_{0}}(\cdot)\right) \\
\leqq & \bar{\gamma}_{n} \frac{1-\left(\gamma_{n}-\bar{\gamma}_{n}\right)^{n^{u}}}{1-\left(\gamma_{n}-\bar{\gamma}_{n}\right)}+\left(\gamma_{n}-\bar{\gamma}_{n}\right)^{n^{u}},
\end{aligned}
$$


where

$$
\begin{gathered}
\bar{\gamma}_{n}=1-\exp \left(-\frac{\text { const }}{n^{t / 2}}\right), \\
\gamma_{n}=1-\exp \left(-p(n)^{2-\alpha}\right), \\
p(n)=[(u+1) \ln n]^{2 / 1+\delta / 2},
\end{gathered}
$$

and

$$
\max _{\sigma_{V_{i}}} f_{i}\left(\sigma_{V_{\imath}}\right) / \min _{\sigma_{V_{i}}} f_{i}\left(\sigma_{V_{\imath}}\right) \leqq \exp \left(2 p(n)^{2-\alpha}\right)
$$

with the same probability. With this choice of the functions $\gamma(n), \bar{\gamma}(n), p(n)$ for $n$ sufficiently large, we obtain the result of the lemma.

Consider now a block $B(n)$ and let $\bar{\alpha}_{n}, \bar{\alpha}_{n-1}$ be the spin configurations of the two adjacent $A$-blocks and define:

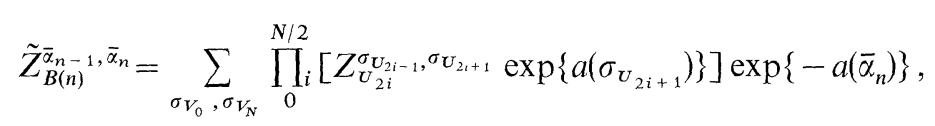

where

$$
\begin{gathered}
\bigcup_{1}^{N} U_{i}=B(n), \quad U_{i} \cap U_{i+1}=\emptyset, \quad\left|U_{i}\right|=q_{0}\left[\left(\log _{+} n\right)^{\varrho(1-\mu)}-\frac{1}{2}\right], \\
N=2\left[\left(\log _{+} n\right)^{\varrho \mu}\right], \quad \sigma_{U_{-1}}=\bar{\alpha}_{n-1}, \quad \sigma_{U_{N+1}}=\bar{\alpha}_{n} .
\end{gathered}
$$

Since $\tilde{Z}_{B(n)}^{\bar{\alpha}_{n-1}, \bar{\alpha}_{n}}$ is obtained by $Z_{B(n)}^{\bar{\alpha}_{n-1}, \bar{\alpha}_{n}}$ switching off the interactions between nonadjacent $U_{i}$ 's it follows from Lemma 2.1 that

$$
\exp \left\{-\frac{\operatorname{const}\left(\log _{+} n\right)^{\varrho \mu}}{q_{0}^{\lambda}\left(\log _{+} n\right)^{\varrho(1-\mu) \varepsilon}}\right\} \leqq \frac{\tilde{Z}_{B(n)}^{\bar{\alpha}_{n-1}, \bar{\alpha}_{n}}}{Z_{B(n)}^{\bar{\alpha}_{n-1}, \bar{\alpha}_{n}}} \leqq \exp \left\{+\frac{\operatorname{const}\left(\log _{+} n\right)^{\varrho \mu}}{q_{0}^{\lambda}\left(\log _{+} n\right)^{\varrho(1-\mu) \varepsilon}}\right\}
$$

with probability $\geqq 1-\exp \left\{-\operatorname{const} q_{0}^{1+2 \delta}\left(\log _{+} n\right)^{\varrho(1-\mu)}\right\}\left(\log _{+} n\right)^{\varrho \mu}$. Notice that $\mu<(1-\mu) \varepsilon$ implies that $Z_{B(n)}^{\bar{\alpha}_{n-1}, \bar{\alpha}_{n}} \sim \tilde{Z}_{B(n)}^{\bar{\alpha}_{n-1}, \bar{\alpha}_{n}}$ for $n$ sufficiently large.

Let us consider the following event $\exists k: 1<k<N$,

$$
Z_{U_{k}}^{\sigma_{U_{k-1}} \sigma_{U_{k+1}}}=\frac{Z_{U_{k}}^{\sigma_{U_{k-1}}, 0} Z_{U_{k}}^{0, \sigma_{U_{k+1}}}}{Z_{U_{k}}^{0,0}} \exp \left\{\eta\left(\sigma_{U_{k-1}}, \sigma_{U_{k+1}}\right)\right\}
$$

with

$$
\sup _{\sigma, \sigma^{\prime}}\left|\eta\left(\sigma, \sigma^{\prime}\right)\right| \leqq \frac{1}{\left[q_{0}\left(\log _{+} n\right)^{\varrho(1-\mu)}\right]^{\varepsilon / 4}}=\bar{\eta}
$$

If this event is verified we have:

$$
\exp \{-4 \bar{\eta}\} \leqq \frac{\tilde{Z}_{B(n)}^{\bar{\alpha}_{n-1}, \bar{\alpha}_{n}} \tilde{Z}_{B(n)}^{0,0}}{\tilde{Z}_{B(n)}^{\bar{\alpha}_{n-1}, 0} \tilde{Z}_{B(n)}^{0, \bar{\alpha}_{n}}} \leqq \exp (+4 \bar{\eta})
$$


If we notice that when such a $k$ does not exist, then for every block $U_{i}$ $(i=1,2, \ldots, N)$ the following inequality should be verified for some $\sigma_{U_{2 i-1}}, \sigma_{U_{2 i+1}}$ :

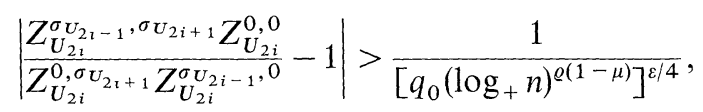

so that by Lemma A.1 it easily follws that:

$$
\mathbb{P}\left(\left|\psi\left(\alpha_{i}, \alpha_{i+1}\right)\right|>\frac{1}{\left[q_{0}\left(\log _{+} n\right)^{\varrho(1-\mu)}\right]^{\varepsilon / 4}}\right) \leqq\left[\frac{1}{q_{0}\left(\log _{+} n\right)^{\varrho(1-\mu)}}\right]^{(\ln n)^{\varrho \mu}}
$$

then by choosing $\mu=\frac{\varepsilon / 2}{1+\varepsilon}$ we conclude the proof.

\section{Appendix B. Proof of Lemma 3.1}

We start from the equality

$$
\begin{aligned}
D^{k} & \sum_{\substack{S_{1} \ldots S_{n} \subset \mathbb{Z} \\
\left(S_{1} \ldots S_{n}\right) \cap T \neq \emptyset}} \varphi\left(S_{1} \ldots S_{n}\right) \prod_{i=1}^{n} \Theta\left(S_{i}\right) \\
= & \sum_{\substack{S_{1} \ldots S_{n} \subset \mathbb{Z} \\
\left(S_{1} \ldots S_{n}\right) \cap T \neq \emptyset}} \varphi\left(S_{1} \ldots S_{n}\right) \sum_{k_{1}+\ldots+k_{n}=k} \frac{k !}{k_{1} ! \ldots k_{n} !} \prod_{i=1}^{n} D^{k_{i}} \Theta\left(S_{i}\right) .
\end{aligned}
$$

Recalling definition (2.36), we have for $S=D_{1} \ldots D_{m},|\tilde{S}|=p$ :

$$
\begin{aligned}
D^{k} \Theta(S)= & \sum_{\alpha \tilde{s}} \sum_{k_{1}+\ldots+k_{p+m}} \frac{k !}{k_{1} ! \ldots k_{p+m} !} \prod_{i=1}^{p} D^{k_{i}}\left(\frac{\exp \left[\tilde{a}\left(\alpha_{n_{i}}\right)\right]}{\sum_{\alpha_{n_{i}}} \exp \left[\tilde{a}\left(\alpha_{n_{t}}\right)\right]}\right) \\
& \cdot \prod_{j=1}^{m} D^{k_{j+p}}\left(\exp \left[V_{D_{j}}\right]-1\right) .
\end{aligned}
$$

Now calling $n_{D}=\sup _{Q(n) \in D}|n|$, suppose we are able to prove the following estimate:

$$
\left\|D^{k} \exp \left[V_{D}\right]\right\| \leqq C_{k}^{1}\left(\log _{+} n_{D}\right)^{\varrho k} \bar{V}_{D}
$$

for some constant $C_{k}^{1}$ depending only on $k$ and for suitable $\bar{V}_{D}$, then, since the following inequality

$$
\left|\sum_{\alpha_{m}} D^{k}\left(\frac{\exp \left[\tilde{a}\left(\alpha_{m}\right)\right]}{\sum_{\alpha_{m}} \exp \left[\tilde{a}\left(\alpha_{m}\right)\right]}\right)\right| \leqq C_{k}^{2}\left(\log _{+} m\right)^{\varrho k}
$$

is easily seen to be true for some constant $C_{k}^{2}$, we can write:

$$
\left|D^{k} \Theta(S)\right| \leqq(p+m)^{k}\left(\log _{+} n_{S}\right)^{2 \varrho k} C_{k} \prod_{D \subset S} \bar{V}_{D},
$$

where

$$
n_{S}=\sup _{D \subset S} n_{D}, \quad C_{k}=\left[\left(\sup _{j \leqq k} C_{j}^{1}\right)\left(\sup _{j \leqq k} C_{j}^{2}\right)\right] .
$$


Now since each $S$ is connected and the set $S_{1} \ldots S_{n}$ forms a cluster [i.e. it cannot be separated into two nonoverlapping parts, otherwise $\left.\varphi\left(S_{1} \ldots S_{n}\right)=0\right]$, and moreover it is supposed to touch the set $T$, we have:

$$
\sup _{i} n_{S_{i}} \leqq n_{T}+\sum_{i=1}^{n} \sum_{D \in S_{i}} d_{D}
$$

where $n_{T}=\sup _{Q(n) \in T}|n|$ and $d_{D}=\max \left(C^{2}, \sup _{Q(n), Q\left(n^{\prime}\right) \in D}\left|n-n^{\prime}\right|\right)$, then

$$
\begin{aligned}
\log \left(\sup n_{S_{\imath}}\right) & \leqq \log \left(n_{T}+\sum_{i=1}^{n} \sum_{D \subset S_{\imath}} d_{D}\right) \leqq \log \left[n_{T} \prod_{i=1}^{n}\left(\prod_{D \subset S_{\imath}} d_{D}\right)\right] \\
& \leqq \log n_{T} \cdot \prod_{i=1}^{n} \prod_{D \subset S_{i}} \log d_{D}
\end{aligned}
$$

so that if for any $k$ a positive constant $\sigma$ is chosen such that:

$$
\sigma^{p+m+1}(p+m)^{k}<1 \quad \forall p, m .
$$

Then if we define

$$
\tilde{\Theta}(S)=\sigma^{|\tilde{S}|} \prod_{D \subset S} \Phi_{D}
$$

with

$$
\Phi_{D}=\frac{\bar{V}_{D}}{\sigma^{2|D| Q^{+2}}}\left[\log d_{D}\right]^{2 \varrho k}
$$

we can write

$$
\begin{aligned}
& \left|D^{k} \sum_{\substack{S_{1} \ldots S_{n} \subset \mathbb{Z} \\
\left(S_{1} \ldots S_{n}\right) \cap T=0}} \varphi\left(S_{1} \ldots S_{n}\right) \prod_{i=1}^{n} \Theta\left(S_{i}\right)\right| \\
& \leqq C_{k}\left(\log _{+} n_{T}\right)^{2 \varrho k} \sum_{\substack{S_{1} \ldots S_{n} \subset \mathbb{Z} \\
\left(S_{1} \ldots S_{n}\right) \cap T \neq \emptyset}}\left|\varphi\left(S_{1} \ldots S_{n}\right)\right| n^{k} \sigma^{n} \prod_{i=1}^{n} \tilde{\Theta}\left(S_{i}\right) \\
& \leqq C_{k}\left(\log _{+} n_{T}\right)^{2 \varrho k} \sum_{\substack{S_{1} \ldots S_{n} \\
\left(S_{1} \ldots S_{n}\right) \cap T \neq \emptyset}}\left|\varphi\left(S_{1} \ldots S_{n}\right)\right| \prod_{i=1}^{n} \tilde{\Theta}\left(S_{i}\right) .
\end{aligned}
$$

Now we have only to prove that the modified potentials $\Phi_{E}$ are well behaved: in other words we want, for $q_{0}$ sufficiently large, the modified activities $\tilde{\Theta}(S)$ to be small enough [cf. Eq. (2.28)] to allow the convergence of the series on the right hand side of Eq. (B.8).

This last requirement is obviously achieved if we can prove that the estimate (B.3) that we have assumed, holds with a $\bar{V}_{D}$ such that:

$$
\sup _{n} \sum_{D \supset A(n)} \Phi_{D} \leqq k\left(q_{0}\right) \underset{q_{0} \rightarrow \infty}{\longrightarrow} 0 .
$$


If condition (B.9) is satisfied we get, by standard methods of the theory of cluster expansion [8]

$$
\sum_{\substack{S_{1} \ldots S_{n} \subset \mathbb{Z} \\\left(S_{1} \ldots S_{n}\right) \cap T \neq \emptyset}}\left|\varphi\left(S_{1} \ldots S_{n}\right)\right| \prod_{i=1}^{n} \tilde{\Theta}\left(S_{i}\right) \leqq|T| \operatorname{const} k\left(q_{0}\right) .
$$

Now for $D=A\left(n_{1}\right), A\left(n_{2}\right), D=A\left(n_{1}\right), Q\left(n_{2}\right)$ it is easy to see that the previous requirements on $D^{k}\left(\exp \left[V_{D}\right]\right)$ can be fulfilled [cf. Eq.(2.35)]. In all the other cases:

$$
V_{D}=\tilde{V}_{D}=\sum_{R_{1} \ldots R_{n}}^{D} \varphi\left(R_{1} \ldots R_{n}\right) \prod_{i=1}^{n} \mathscr{H}\left(R_{i}\right),
$$

to study $D^{k} \tilde{V}_{D}$, we can start by the analogue of Eq. (B.1) and since $\forall L \in \mathscr{F}_{\mathbb{Z}, C}$, for all sufficiently large $q_{0}$ :

$$
\left\|D^{k} \exp \left[W_{L}\right]\right\| \leqq \text { const }\left\|W_{L}\right\|,
$$

then using exactly the same arguments leading to Eq. (B.8), we see that the inequality (B.3) is satisfied with

$$
\bar{V}_{D}=\sum_{R_{1} \ldots R_{n}}^{D}\left|\varphi\left(R_{1} \ldots R_{n}\right)\right| \prod_{i=1}^{n} \tilde{\mathscr{H}}\left(R_{i}\right) .
$$

and

$$
\begin{aligned}
\tilde{\mathscr{H}}(R) & =\tau^{|\tilde{R}|} \prod_{L \in R} \Phi_{L}, \\
\Phi_{L} & =\frac{\|W\|_{L}}{\tau^{4}}\left(\log d_{L}\right)^{2 \varrho k} .
\end{aligned}
$$

The positive constant $\tau$ has to be chosen small enough. Also in this case for $q_{0}$ sufficiently large, condition (B.9) is satisfied so that by Eqs. (B.8), (B.10), and Lemma 3.1 is proven.

Proof of Lemma 3.2. To obtain the proof of Lemma 3.2, which can be considered a corollary of Lemma 3.1, it is sufficient to use the trivial identity:

$$
\begin{aligned}
& \sum_{\substack{S_{1} \ldots S_{n} \subset \Lambda^{\prime} \\
\left(S_{1} \ldots S_{n}\right) \cap A^{\prime} \backslash A \neq \emptyset \\
\left(S_{1} \ldots S_{n}\right) \cap T \neq \emptyset}} \varphi\left(S_{1} \ldots S_{n}\right) \prod_{i=1}^{n} \Theta\left(S_{i}\right) \\
= & \sum_{\substack{S_{1} \ldots S_{n} \subset A^{\prime} \\
\left(S_{1} \ldots S_{n}\right) \cap A^{\prime} \backslash A \neq \emptyset \\
\left(S_{1} \ldots S_{n}\right) \cap T \neq \emptyset}}\left(\prod_{i=1}^{n} \frac{1}{\left(d_{S_{i}}\right)^{1 / 4}}\right) \varphi\left(S_{1} \ldots S_{n}\right) \prod_{i=1}^{n} \tilde{\Theta}\left(S_{i}\right),
\end{aligned}
$$

where $\bar{\Theta}(S)=\Theta(S) d_{S}^{1 / 4} ; d_{S}=\sup _{Q(n), Q\left(n^{\prime}\right) \in S}\left|n-n^{\prime}\right|$ and remark that for every cluster of polymers $S_{1} \ldots S_{n}$ :

$$
\left(S_{1} \ldots S_{n}\right) \cap \Lambda^{\prime} \backslash \Lambda \neq \emptyset, \quad\left(S_{1} \ldots S_{n}\right) \cap T \neq \emptyset,
$$

one has

$$
\prod_{i=1}^{n} \frac{1}{\left(d_{S_{\imath}}\right)^{1 / 4}} \leqq \frac{1}{(d(T, \partial \Lambda))^{1 / 4}}
$$


Then it is quite simple to generalize Lemma 3.1 to the system of polymers with activity $\bar{\Theta}$ for $q_{0}$ sufficiently large.

Acknowledgements. We wish to thank Giovanni Gallavott1 and Errico Presutti for very helpful discussions and comments.

\section{References}

1. Cassandro, M., Olivieri, E.: Commun. Math. Phys. 80, 255 (1981)

2. Khanin, K.M., Sinai, Ya.G.: J. Stat. Phys. 20, 573 (1979)

3. Khanin, K.M.: Theor. Mat. Fiz. 43, 253 (1980)

4. Griffiths, R.B., Lebowitz, J.L.: J. Math. Phys. 9, 1284 (1968)

5. Griffiths, R.B.: Phys. Rev. Lett. 23, 17 (1969)

6. Georgii, H.O.: J. Stat. Phys. 25, 369 (1981)

7. Ibragimov, I.A., Linnick, Yu.V.: Independent and stationary random variables. Groeningen: Walter Noordholf Publishing Co. 1971

8. Gallavotti, G., Martin-Lof, A., Miracle-Sole, S.: In: Lectures Notes in Physics, Vol. 20, pp. 162. Berlin, Heidelberg, New York: Springer 1971

9. Dyson, F.: Commun. Math. Phys. 12, 91 (1969)

10. Ruelle, D.: Commun. Math. Phys. 9, 267 (1968)

11. Dobrushin, R.L.: Teor. Ver. Ee Primen. 13, 201 (1968)

Communicated by T. Spencer

Received May 19, 1982 